\title{
Analysis of the Clinical Significance of the Blood Lipid Test in Patients with Diabetes
}

\author{
Yongcun Huang ${ }^{1, a}$, Wentao Huang ${ }^{2,}$, , Ling $\mathrm{Yu}^{3}$ \\ ${ }^{1}$ Department of Laboratory, The Second Affiliated Hospital of Mudanjiang Medical University, \\ Mudanjiang, 157011, China; \\ ${ }^{2}$ Department of Laboratory, Hongqi Hospital, Mudanjiang Medical University, Mudanjiang, \\ 157011,China; \\ ${ }^{3}$ Department of Neurology, Mudanjiang City Second People's Hospital Brain Branch, Mudanjiang, \\ 157000,China; \\ asdopp18@163.com
}

*Corresponding author huang123@126.com

Keywords: Blood lipid test; Diabetes; Diagnosis; Meaning

\begin{abstract}
Objective: To observe and analyze the application value in clinical diagnosis of the diabetes patients with blood lipid inspection. Methods: From June 2013 to June 2014 we select 50 patients with diabetes and 50 healthy people in physical examination as the research object, they can be divided into the observation group and the control group, two groups were taken an empty stomach blood test in the early morning, and determination of TG, TC, LDL and HDL content of indicators, and to test two groups' blood lipid content. Results: The observation group of patients with blood lipid content is significantly higher than the control group in the crowd of lipid content, and examined the blood lipid levels after contrast; it is the statistically significant difference (P < 0.05). Conclusions: The clinical blood lipids in patients with diabetes can help physicians for patients with complications secondary to the timely and effective diagnosis, treatment and prevention, so at the time of diagnosis and treatment in patients with diabetes, in addition to reduce the patient's blood sugar, also to pay attention to the technology of monitoring the patient's blood lipid levels.
\end{abstract}

\section{Introduction}

The section headings are in boldface capital and lowercase letters. Second level headings are typed as part of the succeeding paragraph (like the subsection heading of this paragraph).

The diabetes is a common endocrine disease, patients with syndrome of the atherosclerosis. Seriously ill patients have micro vascular and large vascular lesions. Diabetes, insulin resistance, that is, patients with pancreatic islet beta cells in the body is not sensitive to insulin. Diabetes patients in general are obese, lipoprotein lipase activity, their higher than healthy people. Due to the increasing the concentration of plasma free fatty acids and TG, are caused fat metabolism. Blood lipid test is of great significance to the clinical diagnosis of diabetic patients; in the treatment of atherosclerosis has all the application and promotion. After a long period of clinical practice has proved that in the treatment of 50 cases of patients with diabetes, blood lipid, inspection, the curative effect of concrete, are reported as follows.

\section{Materials and Methods}

\section{Materials}

We select our hospital from June 2013 to June 2014 were the clinical data of 50 cases of patients with diabetes, patients can be divided into observation group and 30 cases of male patients, 20 patients with female, aged between 38 to 78 years, average age $(55.6 \pm 10.5)$, the average duration in five years. Selection in the same period of 50 cases of blood sugar normal persons as control group. 
The 29 patients with male, female patients with 21 cases. Two groups of patients' age, weight, alcohol, tobacco, hobby and general data, such as the pressure of work, there was no statistically significant difference $(\mathrm{P}>0.05)$, and can be used as a contrast.

\section{Methods}

1) Blood collection. Since the study will have to use blood samples, so the observation group and control group two groups of 3 weeks before blood samples all should keep good habits, do not take drugs affect blood lipid index, began a fast, and the day before blood samples in time mouth blood samples early in the morning, each $3 \mathrm{ml}$ venous blood and serum separation, made within the prescribed time of three phthalein glycerin (TG), total cholesterol (TC), low density lipoprotein (LDL) and high-density lipoprotein (HDL) index of content determination of the work.

2) The instruments and reagents used American Beckman company AU680 automatic biochemical analyzer for testing. Study used reagents mainly come from the original reagent Beckman Company, within the scope of all reagents in the period of validity.

3) inspection methods using enzymatic TG and TC index content; Using two point terminal method inspection index of LDL and HDL levels.

\section{Statistical processing}

With mathematical statistics software SPSS19.0 for data collection and statistical analysis, and chi-square test, $\mathrm{P}<0.05$, significant difference has statistical significance.

\section{Results}

The observation group of patients with blood lipid content is significantly higher than control group in the crowd of blood lipid content, examined the blood lipid levels after contrast, statistically significant difference $(\mathrm{P}<0.05)$.

Table 1: Each index of two groups of blood lipid content detection

\begin{tabular}{lllll}
\hline Group & TG & TC & LDL-C & HDL-C \\
\hline $\begin{array}{l}\text { The } \\
\text { observation } \\
\text { group }\end{array}$ & $2.76 \pm 1.18$ & $5.32 \pm 1.12$ & $3.36 \pm 1.08$ & $1.86 \pm 0.62$ \\
$\begin{array}{l}\text { The control } \\
\text { group }\end{array}$ & $1.57 \pm 0.83$ & $4.13 \pm 0.75$ & $2.74 \pm 0.91$ & $1.52 \pm 0.57$ \\
$\chi 2$ & 6.058 & 7.621 & 6.895 & 6.023 \\
P value & $<0.05$ & $<0.05$ & $<0.05$ & $<0.05$ \\
\hline
\end{tabular}

\section{Discussion}

The epidemiological investigation, according to the results of patients with diabetes can appear not harmonious phenomenon of lipid metabolism, and even induce hyperlipidemia. The studies have confirmed that diabetic dyslipidemia is complicated by a major risk factor for cardiovascular complications, the existence of hyperlipidemia diabetes disease progression. In the study of blood lipid test in the clinical diagnosis of patients with diabetes to evaluate the value of the purpose of the analysis, for the same period of diabetic patients admitted in the hospital and health physical examination for blood testing, the indicators in the by contrast, found that patients with diabetes mellitus group TG, TC, LDL - C level significantly increased compared with healthy controls, the level of HDL - C significantly reduced, and the results are consistent with literature reports the results. The function of insulin is diversiform, can make high lipoprotein lipase activity increases, to control the activity of hormone sensitive lipase, very low density lipoprotein cholesterol and insulin in liver synthetic function plays an important regulatory role. Insulin action happens disorder, is 
bound to a certain degree of influence on the human body lipid levels. Epidemiological survey, the world will be more than $50 \%$ in patients with diabetes risk exists abnormal lipid protein, therefore, to prevent the clinical value of diabetes complicated with dyslipidemia. It is important to note that lipoprotein hematic disease diabetes mellitus patients with secondary and primary two kinds, single blood lipid test was performed at for diabetes patients with abnormal lipoprotein levels, the identification accuracy is not high, blood lipid indicators comprehensive detection has more important meaning for diabetes treatment and observation. Lipid metabolism function in patients with diabetes is not harmonious, especially not insulin-dependent diabetes complications of patients with hyperlipidemia. Only for blood sugar control, scientifically adjust patients' plasma lipoprotein.

\section{The diagnostic value of the total cholesterol (TC)}

The function of insulin is varied, it not only can improve the activity of lipoprotein lipase and conducive to control the activity of hormone sensitive lipase, but also in the largest extent, it improves the liver function of very low density lipoprotein cholesterol synthesis. In regulating lipid and lipoprotein metabolism, etc., the influence of insulin plays an important role. In diabetic patients according to the statistics, the world will be more than half of the patients can appear the possibility of abnormal lipoprotein hematic disease; about $75 \%$ of patients appear particularly high risk of type IV high lipoprotein hematic disease. According to related reports, diabetes associated with secondary abnormal lipoprotein hematic disease probability of $72 \%$, so we must take timely treatment of diabetes complicated with abnormal lipoprotein hematic disease prevention measures, does not cause symptoms of diabetes mellitus complicated with coronary heart disease (CHD). Diabetic patients with abnormal lipoprotein hematic disease, on the other hand, it contains the secondary and primary. Single lipid assay, in patients with diabetes abnormal lipoprotein levels, including secondary and primary identification accuracy is not high; its main function is to observe diabetes treatment response.

Because of the influence of insulin can be through a variety of ways and means and regulating lipid and lipoprotein metabolism, according to statistics, about $40 \%$ of patients with diabetes complicated by abnormal lipoprotein hematic disease, about $80 \%$ of them for high triglyceride levels, namely the $\mathrm{W}$ high lipoprotein hematic disease. Epidemiological investigation and study, found that diabetes mellitus accompanied by secondary abnormal lipoprotein hematic disease patients than in patients with concurrent three times higher incidence of coronary heart disease (CHD), so the effective prevention and treatment of diabetes complicated with abnormal lipoprotein hematic disease is one of the keys to reduce diabetes mellitus complicated with coronary heart disease (CHD). It is important to note that not abnormal lipoprotein hematic disease occurred in patients with diabetes mellitus are secondary, some may be diabetes complicated with primary abnormal lipoprotein hematic disease. Pure blood lipid test is very difficult to complete the identification of the two, the main differential response to diabetes treatment or observation.

\section{The diagnostic value of the triglycerides (TG)}

The insulin resistance and glucose metabolism function in patients with diabetes are not stable; it has the characteristics of the secondary. Only scientifically adjust blood sugar, can inhibit the increase of cholesterol. Due to all the length of the course of the disease and lack of insulin level in patients with completely different, after careful observation in long-term clinical practice, found that high blood triglycerides (TG) and the correlation between insulin resistance is very big. Most of the insulin resistance syndrome in patients with TC levels is higher than healthy people. Relatively, triglyceride (TG) island, hematic disease, more obese patients plasma insulin level is high. At the same time, the insulin resistance syndrome will increase the role of atherosclerosis in the largest degree. Triglyceride (TG) of diabetes patients with highly if severe, then his triglyceride composition proportion of low density lipoprotein cholesterol is higher.

Due to different duration, and degree of insulin deficiency, there are more studies observed high TG levels, and insulin resistance (IR) syndrome between very close relationships. In people with type 1 diabetes (T2DM) B is often the cause of excessive endogenous insulin secretion of hyperinsulinemia is to compensate the original insulin resistance, most patients with insulin 
resistance syndrome combined TG levels. Equally high TG levels, some patients with obesity and elevated plasma insulin levels at the same time, more importantly, can also cause insulin resistance syndrome LDDC structure is unusual, with high TG levels, exist at the same time, have a strong role of atherosclerosis. TG and VLDL (50\%-100\%) of the type 2 diabetes will be a moderate increase, particularly in obese patients, may be due to VLDI. And apoB100 synthesis, level of plasma LDL - C is usually normal, but triglycerides, LDL - C. HDL - C will usually reduce the richest triglycerides.

\section{The cholesterol effect of low density lipoprotein cholesterol (LDL) and high-density lipoprotein (HDL) on the diabetes}

Because the patient's length of the course of the disease and the degree of insulin deficiency are completely different, so the patient also has the obvious difference between plasma lipoprotein levels. Research data show that patients with good blood sugar control, they can't high and low density lipoprotein, but out of more normal and stable level. On the other hand, patients with poor blood sugar control, and to compare the lack of insulin level, resulting in high blood triglycerides and hypercholesterolemia chance is very high.

HDL and LDL - C - C with diabetes relationship analysis because of the different duration, and degree of insulin deficiency patients' plasma lipoprotein levels can have very big difference. Studies have shown that in patients with good blood sugar control, very low density lipoprotein (VLDL) and low density lipoprotein (LDL - C) in normal or low level, HDL - C can be at a higher level, and poor blood sugar control of patients is due to the lack of insulin, prompted the liver not only generate increased VLDL, giant because of lipoprotein (LPL) activity of cool decreased, decrease in VLDL clearance, so often appear high blood triglyceride vinegar and hypercholesterolemia, and diabetes ketosis can appear even particles.

The diabetes is by genetic and environmental factors interact and lead to the question of a group of metabolic abnormalities syndrome. Can lead to a patient's body multiple system organs in chronic complications, clinical morbidity and mortality in patients with the disease is very high. With the continuous development of today's society economy, people's living standards are constantly improve, due to the accelerating pace of life, in People's Daily protein too much food of high quantity of heat, and people are engaged in manual Labor, less energy consumption, resulting in the phenomenon of dyslipidemia and obesity has become a common phenomenon in society at present. According to the clinical investigation and study, Diabetes can lead to the whole body organs, chronic complications of multiple system exist relatively high morbidity and mortality. Current clinical many studies have shown that patients with type 2 diabetes is often accompanied by symptoms such as obesity, hyperlipidemia and hypertension, is the so-called metabolic syndrome, a recent period of time of clinical meta-analysis results showed that the metabolic syndrome is cardiovascular events is a major risk factor for increased significantly. To sum up, for the crowd of dyslipidemia, the probability of the diabetes were significantly higher than that of blood lipids, therefore, the prevention and treatment of diabetes in the process of dealing with blood lipid test attach importance to it, can get timely diagnosis and treatment of diabetes.

The diabetes is by a variety of pathogenic factors, such as genetic factors, immune disorders, microbial infection and its toxin, mental factors acting on the body reduced islet function, insulin resistance that results in sugar, fat, protein, water and electrolyte, and a series of metabolic disorder syndrome, high blood sugar as the main clinical features. Diabetes sugar metabolic disorder will further lead to fat metabolism disorder, at the same time, the abnormal metabolism of blood fat can affect glucose metabolism, disease progression in patients with diabetes, and both influence each other.

In recent years, with the continuous improvement of people's living standard, the ceaseless improvement of diet, the incidence of diabetes in increasing. Diabetes has become one of the major chronic diseases of serious threat to people health. Diabetes mainly by insufficient insulin secretion, insulin resistance or lack of several major causes, causing metabolic disorder syndrome in patients, the main symptoms for sugar metabolic disorder, clinical mainly for hyperglycemia continue to rise. 
Through the study concluded that, to test for diagnosis of diabetes, blood lipid levels, according to the inspection results indicate early not present all kinds of complications in type 2 diabetic patients, need to be in control, strengthen the control diet and living habits, reduce the incidence of coronary heart disease, reduce the pressure for treatment and control the deterioration of disease. Most patients with insulin resistance of TG and TC levels are higher than that of healthy people, and his triglycerides level is to a certain extent, improve the effect of patients with atherosclerosis. At the same time, according to the total cholesterol levels and the patient's physical labor and growth phase correlation, the bad border can be controlled by strengthening the patient's physical exercise. Lipoprotein cholesterol levels of patients show at the same time, the results show that the team LDL levels are significantly higher than the control group, the content of HDL levels significantly lower than the control group, suggests that the metabolism of patients with more severe influence the lipoprotein cholesterol levels. When the client to find out the abnormal situation level, can be controlled by drug treatment, and patients in the treatment process need to strengthen the control, such as alcohol, tobacco, inhibit cholesterol in patients with rising. And had longer duration of diabetes appear more, in the process of treatment to insulin dependence is also different, so the present different plasma lipoprotein levels, namely the diabetes patients in the treatment of effective control of blood sugar, in the process of its performance of HDL, LDL won't appear all kinds of high on the low side. Higher TC levels can lead to high blood pressure; a high level of TU is one of the important risk factors for cardiovascular disease; Higher LDL - C is a major risk factor for the occurrence and development of atherosclerosis. The low level of HDL - $\mathrm{C}$ is a harbinger of coronary heart disease, clinical and can promote the development of atherosclerosis.

The insulin has various functions and to improve lipoprotein lipase activity, effectively control the activity of hormone sensitive lipase, very low density lipoprotein cholesterol synthesis of liver function, is conducive to regulating lipid and lipoprotein metabolism, etc. Diabetes of lipoprotein lipase activity is a important factor which causes the mechanism of diabetic dyslipidemia, gene expression and insulin action determines the activity of lipoprotein lipase, continue to fall as the insulin level in diabetic patients, reduced insulin action, in the largest extent, reduces the activity of lipoprotein lipase abnormal lipoprotein hematic disease, patients with type 2 diabetes. Need to take timely prevention and treatment measures, avoid the occurrence of diabetes mellitus complicated with coronary heart disease (CHD).

\section{Conclusion}

This data shows that the clinical blood lipids in patients with diabetes. It helps the physicians for patients with complications secondary to the timely and effective diagnosis, treatment and prevention. At the time of diagnosis and treatment in patients with diabetes, in addition to reducing the patient's blood sugar, blood lipid levels, also paying attention to technology to monitor patients.

\section{Acknowledgement}

This research was financially supported by the National Science Foundation.

\section{References}

[1] Eoin P.O' Sullivan, David T. Ashley, Colin Davenport, Niamh Devlin, Rachel Crowley, Amar Agha, Christopher J. Thompson, DonalO' Gorman, DiarmuidSmith. Osteoprotegerin and biomarkers of vascular inflammation in type 2 diabetes[J]. Diabetes Metab. Res. Rev. 2010 (6)

[2] Mather, Kieren J,Steinberg, Helmut O,Baron, Alain D. Insulin resistance in the vasculature[J]. Journal of Clinical Investigation. 2013 (3)

[3] Vasudevan A. Raghavan. Insulin Resistance and Atherosclerosis[J]. Heart Failure Clinics. $2012(4)$ 
[4] Ali Ozyol, Oguzhan Yucel, Meltem Refiker Ege,Ali Zorlu, Mehmet Birhan Yilmaz. Microalbuminuria is Associated With the Severity of Coronary Artery Disease Independently of Other Cardiovascular Risk Factors[J]. Angiology . 2012 (6)

[5] Ivana Rumbak, Vesna, Lea Sokoli, Selma Cvijeti, Romana Kajfe, Irena Coli? Bari. Bone mineral density is not associated with homocysteine level, folate and vitamin B12 status[J]. Archives of Gynecology and Obstetrics . 2012 (4)

[6] Meg J Jardine, Amy Kang, Sophia Zoungas, Sankar D Navaneethan, Toshiharu Ninomiya, Sagar U Nigwekar, Martin P Gallagher, Alan Cass,Giovanni Strippoli, Vlado Perkovic. The effect of folic acid based homocysteine lowering on cardiovascular events in people with kidney disease: systematic review and meta-analysis[J]. BMJ. 2012 (2)

[7] Dimitris Tousoulis, Anna-Maria Kampoli, Nikolaos Papageorgiou, Emmanuel Androulakis, haralambos Antoniades, Konstantinos Toutouzas, Christodoulos Stefanadis. Pathophysiology of Atherosclerosis: The Role of Inflammation[J]. Current Pharmaceutical Design. 2011 (37)

[8] G. Togliatto, A. Trombetta, P. Dentelli, A. Rosso, M. F. Brizzi. MIR221/MIR222 -driven post-transcriptional regulation of P27KIP1 and P57KIP2 is crucial for high-glucose- and AGE-mediated vascular cell damage[J]. Diabetologia. 2011 (7)

[9] P. Wiklund,A. Nordstr?m,J.-H. Jansson,L. Weinehall,P. Nordstr?m. Low bone mineral density is associated with increased risk for myocardial infarction in men and women[J]. Osteoporosis International. 2012 (3) 